\title{
Patterns of radiotherapy practice for biliary tract cancer in Japan: results of the Japanese radiation oncology study group (JROSG) survey
}

Fumiaki Isohashi ${ }^{1}$, Kazuhiko Ogawa ${ }^{1 *}$, Hirobumi Oikawa ${ }^{2}$, Hiroshi Onishi ${ }^{3}$, Nobue Uchida $^{4,5}$, Toshiya Maebayashi', Naoto Kanesaka ${ }^{7}$, Tetsuro Tamamoto ${ }^{8}$, Hirofumi Asakura ${ }^{9}$, Takashi Kosugi ${ }^{10}$, Takashi Uno ${ }^{11}$, Yoshinori Ito ${ }^{12}$, Katsuyuki Karasawa ${ }^{13}$, Makoto Takayama ${ }^{14}$, Yoshihiko Manabe ${ }^{15}$, Hideya Yamazaki ${ }^{16}$, Mitsuhiro Takemoto ${ }^{17}$, Yasuo Yoshioka ${ }^{1}$, Kenji Nemoto ${ }^{18}$, Yasumasa Nishimura ${ }^{19}$ on behalf of the Japanese Radiation Oncology study Group (JROSG)

\begin{abstract}
Background: The patterns of radiotherapy (RT) practice for biliary tract cancer (BTC) in Japan are not clearly established.

Methods: A questionnaire-based national survey of RT used for BTC treatment between 2000 and 2011 was conducted by the Japanese Radiation Oncology Study Group. Detailed information was collected for 555 patients from 31 radiation oncology institutions.

Results: The median age of the patients was 69 years old (range, 33-90) and $81 \%$ had a good performance status (0-1). Regarding RT treatment, 78\% of the patients were treated with external beam RT (EBRT) alone, 17\% received intraluminal brachytherapy, and 5\% were treated with intraoperative RT. There was no significant difference in the choice of treatment modality among the BTC subsites. Many patients with EBRT were treated with a total dose of 50 or 50.4 Gy ( 40\%) and only $13 \%$ received a total dose $\geq 60 \mathrm{~Gy}$, even though most institutions (90\%) were using CT-based treatment planning. The treatment field consisted of the primary tumor (bed) only in $75 \%$ of the patients Chemotherapy was used for 260 patients (47\%) and was most often administered during RT (64\%, 167/260), followed by after RT (63\%, 163/260). Gemcitabine was the most frequently used drug for chemotherapy.
\end{abstract}

Conclusions: This study established the general patterns of RT practice for BTC in Japan. Further surveys and comparisons with results from other countries are needed for development and optimization of RT for patients with BTC in Japan.

Keywords: Biliary tract cancer, Radiotherapy, Chemotherapy, Adjuvant, Palliative

\section{Background}

Biliary tract cancer (BTC) is a rare disease that is curable by surgery in fewer than $10 \%$ of all cases. Prognosis depends in part on the anatomic location of the tumor, which affects its resectability. Total resection is possible for $25 \%$ to $30 \%$ of lesions originating in the distal bile duct, a rate that is clearly better than that for lesions in more proximal sites. However, the rate of relapse is as

\footnotetext{
* Correspondence: kogawa@radonc.med.osaka-u.ac.jp

${ }^{1}$ Department of Radiation Oncology, Osaka University Graduate School of Medicine, 2-2 (D-10) Yamadaoka, Suita, Osaka 565-0871, Japan

Full list of author information is available at the end of the article
}

high as 60-75\%, even if clear resection (R0 resection) is possible [1]. In many patients with a tumor that cannot be completely removed by surgery, other treatments such as radiotherapy (RT) or stenting procedures may maintain adequate biliary drainage and improve survival. Optimal management is therefore essential for both postoperative and unresectable BTC.

In Japan, there were an estimated 20,734 new cases of BTC in 2007, with more than a 3 -fold increase over the last three decades [2], while RT has become much more common because new methods and technology for treatment planning are now available. For these reasons, 
optimal management of RT for BTC has become a major concern in Japan. For the study presented here, the Japanese Radiation Oncology Study Group (JROSG) conducted a nationwide questionnaire-based survey on BTC. The questionnaire elicited detailed information regarding patient characteristics, treatment characteristics, and outcomes of treatment. The primary goal of this study was to determine the patterns of RT practice for BTC in order to provide assistance with development of future randomized clinical trials. Therefore, factors influencing the treatment outcome are analyzed elsewhere (Yoshioka et al.: Factors influencing survival outcome in radiotherapy for biliary tract cancer, submitted). To the best of our knowledge, this is the first report to establish how RT is used nationally to treat BTC in Japan.

\section{Methods}

The JROSG conducted a nationwide survey of RT used for BTC treatment between 2000 and 2011 using a questionnaire requesting detailed information on patients and treatment characteristics. Patients were included if they met the following criteria: diagnosis of BTC without evidence of distant metastasis; treatment with RT between 2000 and 2011; no diagnosis of any other malignancy; and no previous RT. Diagnosis of BTC without pathologic verification was based on radiographic findings from contrastenhanced computed tomography (CT), ultrasonography, endoscopic ultrasonography, and endoscopic retrograde/ magnetic resonance cholangiopancreatography.

Of the 71 radiation oncology centers in Japan belonging to the JROSG, 31 (40\%) agreed to participate in the survey. The other centers did not participate mostly because too few BTC patients had been treated with RT at the center in the study period. Each participating center provided a database of patients with BTC treated with RT between 2000 and 2011. The study was performed according to guidelines approved by the institutional review board of each institution whenever necessary.

The Mann-Whitney $U$ test and Student's $t$-test were used to investigate relationships between variables. A $\mathrm{p}$ value of $<.05$ or a $95 \%$ confidence interval not including 1 was considered to be statistically significant. All statistical tests were 2 -sided.

\section{Results}

\section{Data collection}

Detailed information was collected for 555 patients from 31 institutions with a median of 15 patients per institution (range: 1-56 patients). The distribution of the number of institutions based on the number of patients treated between 2000 and 2011 is shown in Figure 1. This indicates considerable variation among institutions in the number of patients treated during the 11-year period: $\leq 10$ patients were treated at 13 institutions

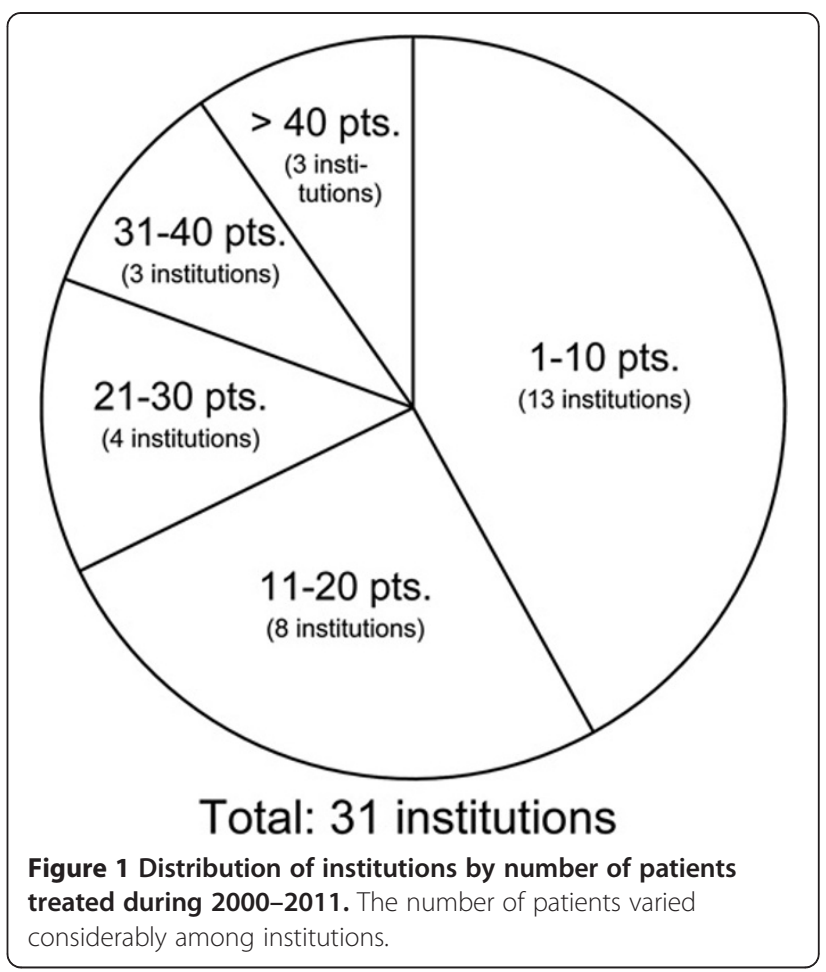

(42\%), while over 30 patients were treated at only 6 institutions (19\%).

\section{Patient and disease characteristics}

The background characteristics of all 555 patients are listed in Table 1. The median age was 69 years old (range, $33-90$ years old) and $48 \%$ of the patients were $\geq 70$ years old. Pre-therapeutic evaluations were performed by ultrasonography, CT, and magnetic resonance cholangiography in $81 \%, 93 \%$, and $58 \%$ of the patients, respectively. Regarding the primary site, $\sim 50 \%$ of BTC lesions arose in the perihilar regions of the extrahepatic bile duct, with distal regions of the extrahepatic bile duct being the second most common site (26\%). Among all patients, $>80 \%$ had an Eastern Cooperative Oncology Group performance status of $0-1, \sim 30 \%$ had a drinking or smoking habit, $52 \%$ had an unresectable tumor at diagnosis, and $53 \%$ had clinical stage T3-4 disease at diagnosis.

\section{Characteristics of surgical procedures}

Primary surgery before RT was performed in 242 patients (44\%). Curative surgery was performed in 235 patients, but only 63 ( $26 \%$ of those who underwent surgery) had complete (R0) resection. R1 resection (microscopic positive margins) and R2 resection (macroscopic residual tumor) were performed in 142 (59\%) and 37 (15\%) patients, respectively. Note that surgeries included non-curative (R2) and curative-intent (R0 or R1) resections, because our cohort was based on a RT database. Lymph node dissection was performed on 
Table 1 Patient and disease characteristics $(n=555)$

\begin{tabular}{l} 
Characteristic \\
\hline Age (median, 69 y) \\
$<70$ y \\
$\geq 70$ y \\
Gender \\
Female \\
Male \\
Pathologic type, verified \\
Yes, adenocarcinoma \\
Yes, other \\
No
\end{tabular}

Ultrasonography (before RT)

Yes

No

Unknown

CT (before RT)

Yes

No

Unknown

MRCP (before RT)

Yes

No

Unknown

PTCD

Yes

No

Unknown

Primary site

Intrahepatic bile duct

Gallbladder

Extrahepatic bile duct

Perihilar

Distal

Unknown

Ampulla of Vater

Maximal tumor size (Median, $4.0 \mathrm{~cm}$ )

$$
\begin{aligned}
& <4.0 \mathrm{~cm} \\
& \geq 4.0 \mathrm{~cm}
\end{aligned}
$$$$
\text { Unknown }
$$

Tumor emboli

$$
\text { Yes }
$$

No

Unknown

ECOG performance status
Patients (\%)

$288(51.9)$
$267(48.1)$
$183(33.0)$
$372(67.0)$

$417(75.1)$

$5(0.9)$

$133(24.0)$

451 (81.3)

21 (3.8)

83 (14.9)

$515(92.8)$
$5(0.9)$
$35(6.3)$

$324(58.4)$

$152(27.4)$

79 (14.2)

242 (43.6)

$151(27.2)$

162 (29.2)

71 (12.8)

42 (7.6)

439 (79.1)

278 (50.0)

$144(25.9)$

17 (3.1)

$3(0.5)$

195 (35.1)

198 (35.7)

$162(29.2)$

$32(5.8)$

$292(52.6)$

$231(41.6)$

(4)

$223(40.2)$
Table 1 Patient and disease characteristics $(n=555)$

(Continued)

\begin{tabular}{cc}
\hline 1 & $226(40.7)$ \\
2 & $77(13.8)$ \\
3 & $17(3.1)$ \\
4 & $1(0.2)$ \\
Unknown & $11(2.0)$ \\
Jaundice &
\end{tabular}

Yes

$355(64.0)$

No or unknown

$200(36.0)$

CA19-9 (U/mL)

$<37$

102 (18.4)

37-1,000

$253(45.6)$

81 (14.6)

$119(21.4)$

Unknown

$300(54.1)$

$<5$

63 (11.3)

49 (8.8)

$\geq 10$

$143(25.8)$

Alcohol consumption

Yes

193 (34.8)

No

$223(40.2)$

Unknown

139 (25.0)

Smoking

Yes

175 (31.5)

No

239 (43.1)

Unknown

141 (25.4)

Diabetes mellitus

Yes

75 (13.5)

No

383 (69.0)

Unknown

97 (17.5)

Clinical T stage

TX

$11(2.0)$

T1

$41(7.4)$

147 (26.4)

183 (33.0)

$112(20.2)$

61 (11.0)

Unknown

310 (55.9)

NO

165 (29.7)

N1

80 (14.4)

Clinical stage

96 (17.3)

202 (36.4) 
Table 1 Patient and disease characteristics $(n=555)$ (Continued)

\begin{tabular}{lc}
\hline III & $146(26.3)$ \\
IV & $25(4.5)$ \\
Unknown & $86(15.5)$ \\
Resectable at diagnosis & \\
Yes & $254(45.8)$ \\
No & $288(51.9)$ \\
Unknown & $13(2.3)$ \\
Investigational protocol & \\
Yes & $0(0)$ \\
No & $555(100)$
\end{tabular}

Abbreviations: RT Radiotherapy; CT Computed tomography; MRCP Magnetic retrograde cholangiopancreatography; PTCD Percutaneous transhepatic cholangiodrainage; ECOG Eastern cooperative oncology group; CEA Carcinoembryonic antigen; CA19-9 Carbohydrate antigen 19-9.

173 patients (71\%) and a positive node was identified pathologically in 85 patients (35\%).

\section{Radiation treatment characteristics}

The most common treatment modality was external beam radiotherapy (EBRT) alone (78\% of the patients), followed by intraluminal brachytherapy (ILBT) with or without EBRT (17\%) and intraoperative RT (IORT) with or without EBRT (5\%). Chemotherapy before, during, or after RT was used for 260 patients (47\%).

The patterns of RT practice or choice of treatment modality according to the BTC subsites are shown in Figure 2. Because the subsites of 17 patients were unknown, the patterns for 538 patients were analyzed. The rate of primary surgery varied according to the tumor subsite: primary surgery was performed for only $30 \%$ of tumors that originated in proximal regions (intrahepatic and perihilar), but for $67 \%$ of those that originated in more distal lesions (distal and gallbladder) $(p<.05)$.
However, there was no significant difference in the choice of treatment modality among the BTC subsites.

Table 2 shows the treatment modality choices according to purpose of RT, which was divided into four groups: RT after curative resection (R0-1) $(\mathrm{n}=183)$, RT after non-curative resection (R2) $(\mathrm{n}=33)$, curative RT for inoperable cases $(n=235)$, and palliative RT for inoperable cases $(n=78)$. The purpose of RT for inoperative cases (curative or palliative) was chosen by radiotherapists who answered the questionnaire. Twenty-six patients with IORT were excluded from this analysis based on a comparison of doses among the variables because strong bias was suspected when a parameter such as IORT was used, which involved a very large dose at one time. Over $90 \%$ of the patients who underwent surgery received EBRT alone. For the patients who did not undergo surgery, there was a tendency for ILBT with EBRT to be used for a curative purpose more often than for a palliative purpose, but the difference was not statistically significant $(25 \%$ vs. $15 \%, p=.08)$. To compare the combined dose of ILBT and EBRT with a single modality dose (ILBT alone or EBRT alone), the total dose (ILBT + EBRT) was calculated as the biologically equivalent dose in 2-Gy fractions $\left(\mathrm{EQD}_{2}\right)$ using the linear quadratic model. The value used for assessing effects on tumors was $\alpha / \beta=10$ Gy. The median $\mathrm{EQD}_{2}$ for EBRT alone, ILBT alone and EBRT with ILBT was $50 \mathrm{~Gy}_{\alpha / \beta 10}, 36 \mathrm{~Gy}_{\alpha /}$ $\beta 10$, and $60 G y_{\alpha / \beta 10}$, respectively, while that for ILBT with EBRT was significantly greater than EBRT alone or ILBT alone $(p=.001)$. In terms of treatment purpose, however, there were no significant differences in the median $\mathrm{EQD}_{2}$ among the groups $(50 \mathrm{~Gy} \alpha / \beta 10$ for all variables).

\section{EBRT characteristics}

The characteristics of the 521 patients who received EBRT are shown in Table 3. The median duration from

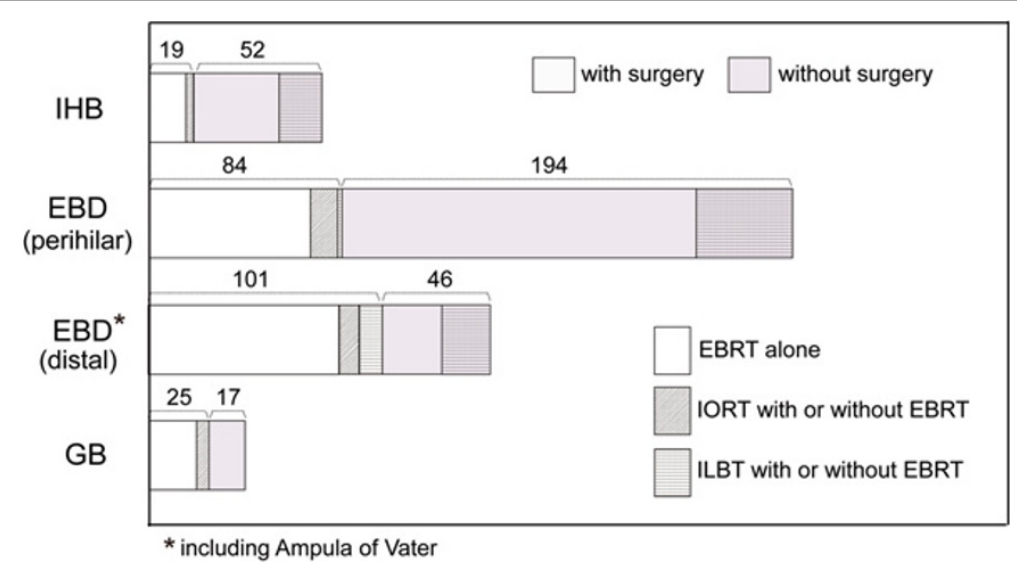

Figure 2 Patterns of radiation practice or choice of treatment modality by BTC subsites. There was no significant difference in the choice of treatment modality among the BTC subsites. 
Table 2 Choices of treatment modality according to purpose of RT ( $n=529$ )

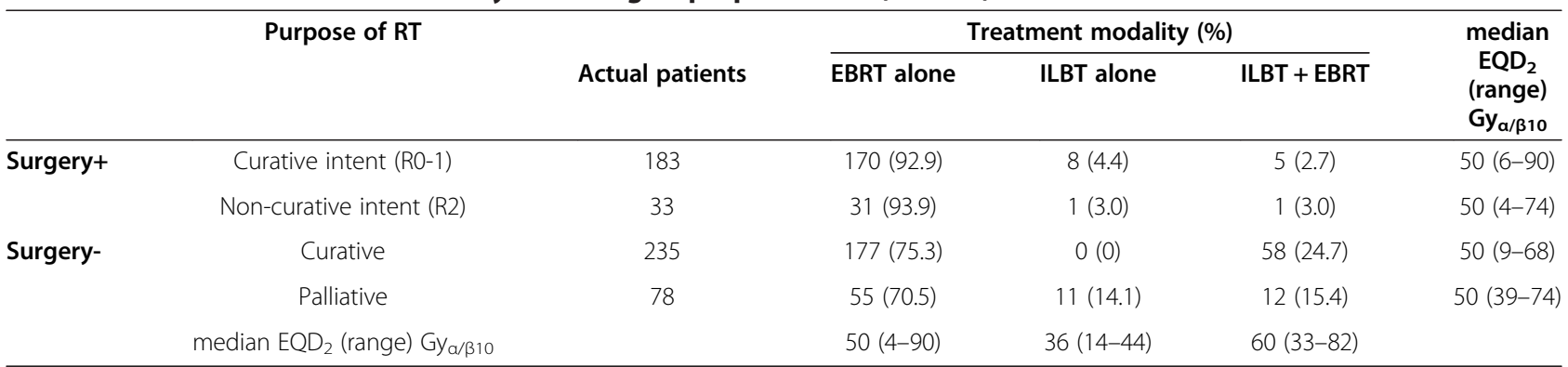

Abbreviations: RT Radiotherapy; EBRT External beam radiotherapy; ILBT Intraluminal brachytherapy; EQD2 The biologically equivalent dose in 2-gray fractions.

Table 3 EBRT characteristics $(n=521)$

\begin{tabular}{lc}
\hline Characteristic & Patients (\%) \\
\hline EBRT Radiation portals & $162(31.1)$ \\
$\quad 2$ portals & $359(68.9)$ \\
$\quad \geq 3$ portals & \\
EBRT beam energy (MV) & $24(4.6)$ \\
$\quad<10$ & $491(94.2)$ \\
$\geq 10$ & $6(1.2)$ \\
Unknown & \\
EBRT dose/fraction (Gy) & $7(1.3)$ \\
$<1.8$ & $131(25.1)$ \\
1.8 & $352(67.6)$ \\
2 & $31(6.0)$ \\
$>2.0$ & \\
EBRT total radiation dose (Gy) & $69(13.2)$ \\
$<40$ & $129(24.8)$ \\
$40-<50$ & $206(39.5)$ \\
$50 / 50.4$ & $52(10.0)$ \\
$>50.4-<60$ & $65(12.5)$ \\
$\geq 60$ &
\end{tabular}

\section{Radiation field}

primary only

primary plus regional LN

LN only

Unknown

CT-based treatment planning

$$
\text { Yes }
$$

No

$388(74.5)$

119 (22.8)

$5(1.0)$

$9(1.7)$

$68(89.8)$

$53(10.2)$

Conformal therapy

Yes

No

Unknown

MRT surgery to EBRT was 34 days (range, 9-88 days). EBRT was administered with $\geq 3$ portals to $69 \%$ of the patients, at $\geq 10$-megavolt beam energy for $>90 \%$, and at 1.8 Gy or 2.0 Gy per fraction; and with a total dose of $\geq 40$ Gy for $\sim 90 \%$. CT-based treatment planning and conformal RT were used for $90 \%$ and $64 \%$, respectively, of patients treated with EBRT, but only two of these patients received intensity-modulated RT (IMRT).

A summary of the EBRT field based on performance of surgery and nodal status is shown in Table 4. The treatment field consisted of the primary tumor only in $388(75 \%)$ of 521 patients and the primary tumor plus regional lymph nodes in 119 (23\%). Patients who underwent surgery received RT for the primary tumor (bed) plus regional lymph nodes more frequently than patients who did not undergo surgery $(29 \%$ vs. $19 \%, p<.01)$. Additionally, among the patients who underwent surgery, RT for the primary tumor (bed) plus regional

Table 4 EBRT field according to performance of surgery and $\mathbf{N}$ stage $(n=521)$

\begin{tabular}{|c|c|c|c|c|}
\hline \multirow[b]{2}{*}{ Group } & \multirow[b]{2}{*}{ Patients (n) } & \multicolumn{3}{|c|}{ Radiation field (\%) } \\
\hline & & Primary & Primary plus LN & Others \\
\hline \multicolumn{5}{|l|}{ Surgery + } \\
\hline Total & 219 & $151(68.9)$ & $63(28.8)$ & $5(2.2)$ \\
\hline pNO & 75 & $54(72.0)$ & $20(26.7)$ & $1(1.3)$ \\
\hline pN1 & 78 & $49(62.8)$ & $29(37.2)$ & $0(0)$ \\
\hline Unknown & 66 & $48(72.7)$ & $14(21.2)$ & $4(6.1)$ \\
\hline $\mathrm{cNO}$ & 111 & 95 (85.6) & $13(11.7)$ & $3(2.7)$ \\
\hline $\mathrm{cN} 1$ & 65 & $43(66.2)$ & $20(30.8)$ & $2(3.0)$ \\
\hline Unknown & 43 & $13(30.2)$ & $30(69.8)$ & $0(0)$ \\
\hline \multicolumn{5}{|l|}{ Surgery - } \\
\hline Total & 302 & $237(78.5)$ & $56(18.5)$ & $9(3.0)$ \\
\hline cNO & 189 & $171(90.5)$ & $11(5.8)$ & $7(3.7)$ \\
\hline $\mathrm{cN} 1$ & 79 & $34(43.0)$ & $44(55.7)$ & $1(1.3)$ \\
\hline Unknown & 34 & $32(94.2)$ & $1(2.9)$ & $1(2.9)$ \\
\hline Total & 521 & $388(74.5)$ & $119(22.8)$ & $14(2.7)$ \\
\hline
\end{tabular}

Abbreviation: EBRT External beam radiotherapy; LN Lymph node. 
lymph nodes of those with clinically positive nodes was more frequently performed than in patients with clinically negative nodes $(31 \%$ vs. $12 \%, p<.01)$. However, patients with pathologically positive nodes tended to receive RT for the primary tumor (bed) plus regional lymph nodes more frequently than patients with pathologically negative nodes, but the difference was not statistically significant ( $37 \%$ vs. $27 \%, p=.16)$. Among patients who did not undergo surgery, RT for the primary tumor and regional lymph nodes of those with clinically positive nodes was more frequently performed compared to patients with clinically negative nodes $(56 \%$ vs. $6 \%$, $p<.01)$. However, some patients with clinically positive nodes also underwent EBRT for the primary tumor only (43\%).

Analyses of practice patterns of EBRT were performed according to caseload of institutions (Figure 3a-d) and patient age (Figure 3e-h). Caseloads were divided into three categories based on the number of patients treated within the study period at each institution $(\leq 10,11-29$, and $\geq 30$ patients). In institutions with $\geq 30$ patients, the rates of postoperative RT (compared to inoperable cases) (Figure 3A), EBRT for the field of the tumor (bed) plus regional LN (compared to tumor only) (Figure 3B),
A
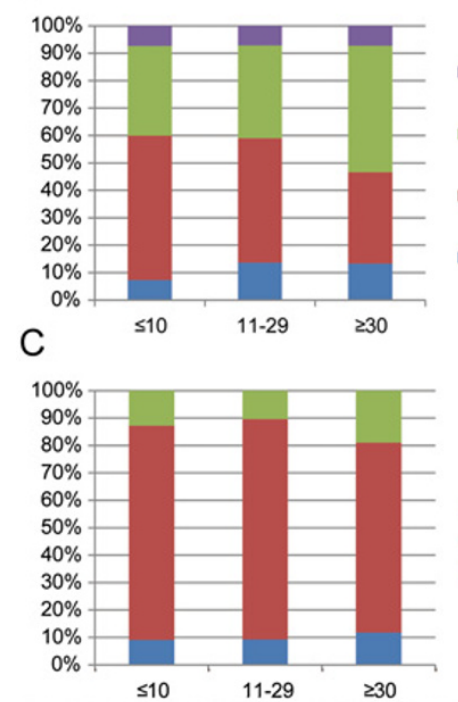

$\overline{\mathrm{E}}$

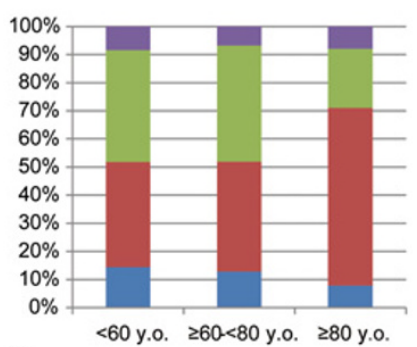

G

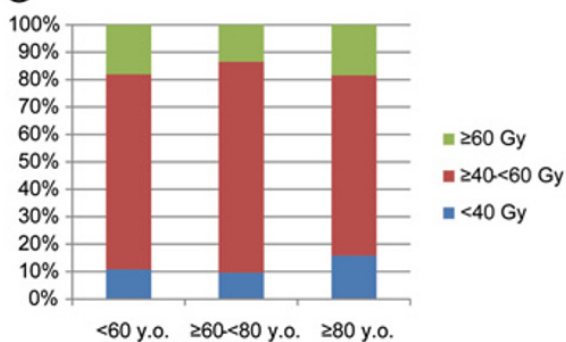

B

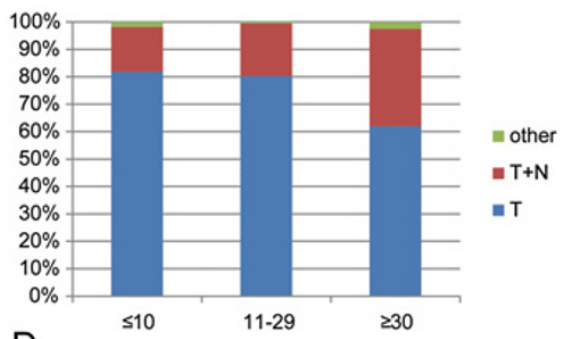

D

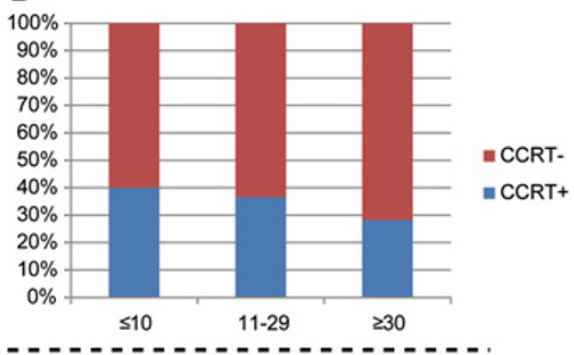

$\mathrm{F}$

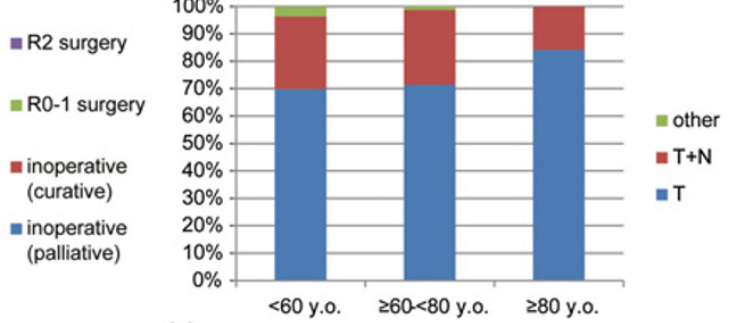

$\mathrm{H}$

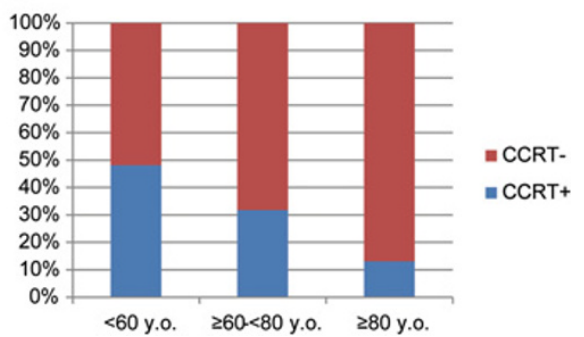

Figure 3 Practice patterns of EBRT according to caseload of institutions or patient age. Three categories were formed based on the number of patients treated at each institution ( $\leq 10,11-29$ and $\geq 30$ patients) $(\mathbf{A}-\mathbf{D})$ or age $(<60, \geq 60-<80$, and $\geq 80$ years old) (E-H) and evaluated based on treatment intent $(\mathbf{A}, \mathbf{E})$, EBRT field size (B, F), EBRT total dose $(\mathbf{C}, \mathbf{G})$, and concurrent chemotherapy $(\mathbf{D}, \mathbf{H})$. 
and patients receiving $\geq 60$ Gy (Figure 3C) were significantly higher than those in institutions with $<30$ patients. Age was also divided into three categories $(<60, \geq 60-<80$, and $\geq 80$ years old $)$. The use of CCRT was significantly higher in patients $<60$ years old compared to those $\geq 60$ - $<80$ years old, and in those $\geq 60-<80$ years old compared to those $\geq 80$ years old (Figure $3 \mathrm{H}$ ).

\section{ILBT and IORT characteristics}

A total of 96 patients (17\%) received ILBT at 13 institutions $(42 \%)$. The characteristics of these cases are listed in Table 5. All 96 patients were treated with ILBT using an iridium-192 source and at 5 or 6 Gy per fraction in $55 \%$ of cases and with a total dose of $\geq 15$ Gy in $85 \%, 76$ (79\%) of whom received ILBT with EBRT at a median EBRT dose of 40 Gy (range, 20-60 Gy). The most common prescription point was $10 \mathrm{~mm}$ from the source (75\%).

IORT was used for only 26 patients $(5 \%)$ at four institutions $(13 \%, 4 / 31), 12(2 \%)$ of whom received IORT with EBRT and 14 (3\%) received IORT alone. The median dose for IORT was 25 Gy (range, 20-30 Gy), with a median beam energy of 12 mega-electron volts (range, 4-25 mega-electron volts).

\section{Chemotherapy}

Chemotherapy was used for 260 patients (46\%), including 167 concurrently with RT (78 concurrently alone; 7

Table 5 Intraluminal brachytherapy $(n=96)$

\begin{tabular}{lc}
\hline Characteristic & Patients (n) \\
\hline Source & \\
$\quad$ Ir-192 & $96(100)$ \\
ILBT single dose/fraction (Gy) & \\
$\quad<5$ & $16(16.7)$ \\
5 & $33(34.4)$ \\
6 & $20(20.8)$ \\
$>6$ & $27(28.1)$ \\
Total dose (Gy) & \\
$<15$ & $14(14.6)$ \\
$15-25$ & $41(42.7)$ \\
$\geq 25$ & $41(42.7)$ \\
Prescription point (from the source) & \\
5 mm & $4(4.2)$ \\
7 mm & $4(4.2)$ \\
10 mm & $72(75.0)$ \\
12 mm & $14(14.6)$ \\
Unknown & $2(2.1)$ \\
With EBRT (Median EQD $2,60.4$ Gy) & $76(79.2)$ \\
Without EBRT (Median EQD $2,35.8$ Gy) & $20(20.8)$ \\
\hline Ab & \\
\hline
\end{tabular}

Abbreviations: Ir Iridium; Gy Gray; EBRT External beam radiotherapy; EQD 2 The biologically equivalent dose in 2-gray fractions.
pre-RT and concurrently; 67 concurrently and post-RT; and 15 pre-RT, concurrently, and post-RT), 4 pre- and post-RT, 12 pre-RT alone, and 77 post-RT alone. The drugs and timing of chemotherapy for these patients are listed in Table 6. Chemotherapy was most often given during RT $(64 \%, 167 / 260)$ followed by after RT $(63 \%$, $163 / 260$ ), while the most frequently used drug for chemotherapy was gemcitabine $(47 \%)$ followed by 5 -FU (37\%). TS-1 and UFT were especially frequently used after RT.

The 167 patients who received chemotherapy during RT (concurrent chemoradiation (CCRT)) were analyzed further because this method has been shown to be efficacious for treatment of patients with BTC with or without surgery. The patients were divided into four groups according to performance of surgery and timing during the study period: Group A, surgery, 2000-2005 ( $n=24)$; Group B, surgery, 2006-2011 ( $\mathrm{n}=30)$; Group C, no surgery, 2000-2005 $(\mathrm{n}=65)$; and Group D, no surgery, 2006-2011 $(n=48)$. There was a significant difference in the use of gemcitabine-containing regimens between Groups A and B and between Groups C and D (Figure 4). This suggests a trend away from the use of 5FU towards a more frequent use of gemcitabine concurrently with RT for patients with BTC treated with or without surgery.

\section{Discussion}

RT for BTC can be classified into adjuvant therapy after surgery or therapy for inoperable cases. While no randomized control trial has been conducted, a meta-analysis revealed that patients with extrahepatic cholangiocarcinoma treated with adjuvant RT show a significantly lower mortality rate than patients treated with surgery alone [3]. Data in the Surveillance Epidemiology and End Result database also suggest that palliative RT prolongs survival in patients with extrahepatic cholangiocarcinoma [4]. In these reports, the outcomes of the treatment were

Table 6 Drugs used and timing of chemotherapy $(n=260)$

\begin{tabular}{lcccc}
\hline Variable & $\begin{array}{c}\text { Actual } \\
\text { patients } \\
\text { (\%) }\end{array}$ & \multicolumn{3}{c}{ Chemotherapy timing (\%) } \\
\cline { 3 - 5 } & 260 & 38 & 167 & 163 \\
\hline Actual patients (n) & & & & \\
Drugs & $122(46.9)$ & $24(63.2)$ & $72(43.1)$ & $78(47.9)$ \\
GEM & $97(37.3)$ & $9(23.7)$ & $74(44.3)$ & $43(26.4)$ \\
5-FU & $40(15.4)$ & $9(23.7)$ & $22(13.2)$ & $15(9.2)$ \\
Cisplatin & $45(17.3)$ & $6(15.8)$ & $5(3.0)$ & $42(25.8)$ \\
TS-1 & $34(13.1)$ & $3(7.9)$ & $12(7.2)$ & $24(14.8)$ \\
UFT & $9(3.4)$ & $3(7.9)$ & $4(2.4)$ & $2(1.2)$ \\
Other & &
\end{tabular}

Abbreviations: RT Radiotherapy; GEM Gemcitabine; 5-FU 5-Fluorouracil; TS-1 Tegafur-gimeracil-oteracil potassium; UFT Tegafur-uracil. 


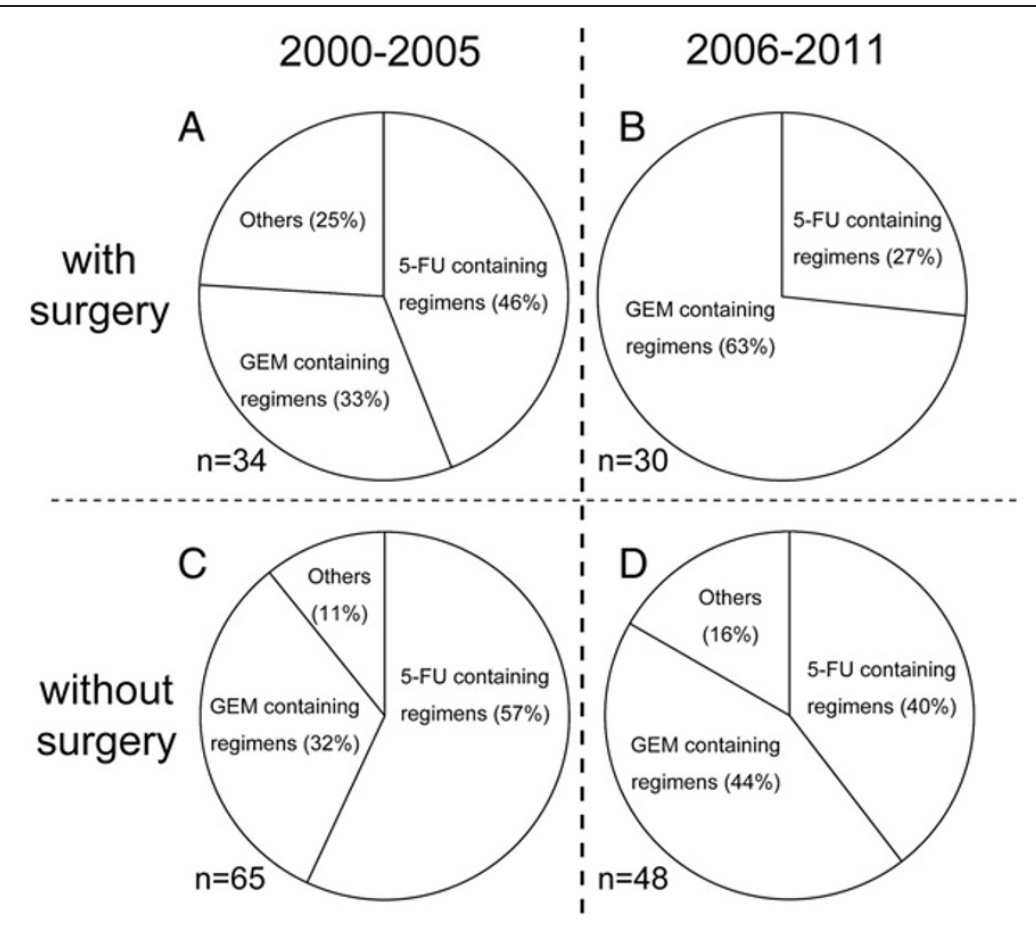

Figure 4 Changes in chemotherapy regime combined with radiotherapy during 2000-2011 based on prior therapy. The patients were divided into four groups according to performance of surgery and the timing during the study period. A: Surgery, $2000-2005$ ( $n=24)$; B: Surgery, 2006-2011 ( $n=30) ;$ C: No surgery, 2000-2005 ( $n=65) ; D$ : No surgery, 2006-2011 $(n=48)$.

reported in detail, but detailed information on RT use has not been provided and there are few reports on patterns of RT practice. We therefore decided to evaluate the practice of RT for BTC at Japanese radiation oncology centers, with the goal of assisting with development of randomized clinical trials. JROSG has conducted similar surveys and successfully determined the general patterns of RT practice for several other cancers in Japan $[5,6]$. Of the 31 responding institutions, $43 \%$ treated fewer than 10 patients over the period covered by the survey. Surprisingly, none of the patients were treated with an investigational protocol, clearly indicating a need for a prospective multicenter study to determine a standard therapeutic approach.

The results of the study showed that CT-based treatment planning was used for approximately $90 \%$ of the patients. Previous nationwide surveys of the structural characteristics of radiation oncology in Japan found that only 329 (45\%) of 726 facilities in 2003 and 407 (57\%) of 712 facilities in 2005 used CT-based treatment planning $[7,8]$. These results suggest that three-dimensional conformal RT planning became mainstream during the survey period or that patients with BTC received RT more frequently in facilities with advanced equipment.

We examined the variations in RT use (modality, total dose, or RT fields) according to the purpose of RT or BTC subsites. Some analyses have suggested that there is a dose-response relationship for treatment of BTC and have stressed the importance of dose escalation $[9,10]$. However, many patients with EBRT included in this survey were treated with a total dose of 50 or 50.4 Gy $(\sim 40 \%)$ and only $13 \%$ of the patients received a total dose $\geq 60$ Gy. These data indicate that use of sufficient doses for EBRT for tumors in the hepatic hilum and liver regions was severely restricted by technical difficulties with the delivery of high doses to these regions while sparing surrounding organs, including the liver, duodenum, stomach, and spinal cord, even though most institutions used CT-based treatment planning. Recently, IMRT has emerged as a sophisticated technique for treatment of tumors, including BTC, in areas at risk of recurrence, while sparing adjacent normal tissue from high-dose irradiation [11]. However, only two patients were treated with IMRT for EBRT during the survey period.

ILBT can also be used for dose escalation in a region at risk $[9,12]$ since it has the advantage of allowing delivery of a sufficient dosage to a target focus while reducing the effect of irradiation on surrounding tissues. Theoretically, a combination of ILBT and EBRT can enhance the beneficial effects of RT, with fewer adverse effects than those incurred with EBRT alone. In fact, ILBT with EBRT entailed a significantly higher $\mathrm{EQD}_{2}$ dose than EBRT alone in our study cohort. While $42 \%$ of the 
institutions performed ILBT, only $14 \%$ of all patients received ILBT combined with EBRT, indicating that this treatment modality was used only in selected cases because the effect of ILBT is limited to the area surrounding the lumen of the biliary tract and improvement in local control can therefore be expected only for small tumors [9].

The optimal radiation field for BTC remains to be defined. The majority of relapses after resection with curative intent occur at the primary tumor site [13], which suggests that it may be reasonable to limit RT to the primary tumor (bed). Only $23 \%$ of the patients included in this survey received radiation to the tumor (bed) as well as the regional lymph nodes, regardless of the lymph node status. Although limiting the radiation field to the tumor (bed) has tended to become prevalent in Japan, the definition of clinical target volume included regional lymph nodes as well as the tumor (bed) in a recent meta-analysis of 14 selected papers with detailed information on adjuvant RT after surgery [3], as well as in many reports on unresectable BTC published since 2000 [14-17]. Collectively, these findings indicate that the radiation field for BTC is not yet standardized due to the lack of a large randomized control trial and that additional studies investigating the optimal radiation field should be conducted.

The study presented here showed that chemotherapy is frequently administered in combination with RT (47\% of all patients). Chemotherapy was most often administered during RT, followed by after RT. Several trials have examined the efficacy of adjuvant chemoradiation after surgery [18] or of chemoradiation for unresectable cases [19]. The National Comprehensive Cancer Network $(\mathrm{NCCN})$ reported that most CCRT for BTC involved the use of 5-FU, and that CCRT with gemcitabine is not recommended due to the limited experience with and potential toxicity of this treatment. However, the use of CCRT combined with gemcitabine-containing regimens increased in Japan during the period covered by the current survey, which suggests that additional studies should be undertaken to establish the optimal sequencing of RT and chemotherapy with drugs such as gemcitabine. For chemotherapy for advanced BTC, the recent randomized control phase III ABC-02 study showed that a combination of gemcitabine and cisplatin improved overall and progression-free survival by $30 \%$ over gemcitabine alone [20]. Based on these results, the combination of gemcitabine and cisplatin can now be considered to be the standard of care as first-line chemotherapy for patients with advanced or metastatic BTC. In Japan, however, oral anticancer drugs such as TS-1 or UFT also tend to be used as adjuvant chemotherapy after RT, and only two patients in the current study were treated with a combination of gemcitabine and cisplatin after RT.

\section{Conclusions}

Patients with BTC should continue to be enrolled in prospective studies of RT with radiosensitizing agents or of RT with dose escalation methods using techniques such as IMRT. Further surveys and comparisons with results from other countries are needed for development and optimization of RT for patients with BTC in Japan.

\section{Consent}

Written informed consent was obtained from the patient for publication of this report and any accompanying images.

\section{Abbreviations}

RT: Radiotherapy; BTC: Biliary tract cancer; EBRT: External beam radiotherapy; JROSG: The Japanese radiation oncology study group; CT: Computed tomography; ILBT: Intraluminal brachytherapy; IORT: Intraoperative radiotherapy; $\mathrm{EQD}_{2}$ : The biologically equivalent dose in 2-Gy fractions; IMRT: Intensity-modulated radiotherapy; CCRT: Concurrent

chemoradiotherapy; NCCN: National comprehensive cancer network.

\section{Competing interests}

The authors made no disclosures and not receive specific funding.

\section{Authors' contributions}

$\mathrm{KO}$ coordinated the entire study. Patient data acquisition was done by $\mathrm{FI}$ $\mathrm{HO}, \mathrm{HO}, \mathrm{NU}, \mathrm{TM}, \mathrm{NK}, \mathrm{TT}, \mathrm{HA}, \mathrm{TK}, \mathrm{TU}, \mathrm{YI}, \mathrm{KK}, \mathrm{MT}, \mathrm{YM}, \mathrm{HY}, \mathrm{MT}, \mathrm{KN}$, and YN. Data analysis was done by $\mathrm{Fl}, \mathrm{KO}$, and $\mathrm{YY}$. The manuscript was prepared by $\mathrm{FI}$. Corrections and/or improvements were suggested by KO and YY. Revisions were done by HO, HO, NU, TM, NK, TT, HA, TK, TU, YI, KK, MT, YM, HY, MT, KN, and $\mathrm{YN}$. All authors read and approved the final manuscript.

\section{Author details}

'Department of Radiation Oncology, Osaka University Graduate School of Medicine, 2-2 (D-10) Yamadaoka, Suita, Osaka 565-0871, Japan. ²Department of Radiology, Iwate Medical University, 19-1 Uchimaru, Morioka, Iwate 020-8505, Japan. ${ }^{3}$ Department of Radiology, University of Yamanashi, 1110, Shimogato Chuo, Yamanashi 409-3898, Japan. ${ }^{4}$ Department of Radiation Oncology, Shimane University, 1060 Nishikawatsu-cho, Matsue-shi, Shimane 690-8504, Japan. ${ }^{5}$ Present affiliation: Department of Radiation Oncology, Tottori Prefectural Central Hospital, 730 Etsu, Tottori-shi, Tottori 680-0901, Japan. ${ }^{6}$ Department of Radiology, Nihon University School of Medicine, 30-1, Ohyaguchi-Kamimachi, Itabashi-ku, Tokyo 173-8610, Japan. ${ }^{7}$ Department of Radiology, Tokyo Medical University, 6-1-1, Shinjuku, Shinjuju-ku, Tokyo 160-8402, Japan. ${ }^{8}$ Department of Radiation Oncology, Nara Medical University School of Medicine, 840 Shijo-cho, Kashihara, Nara 634-8521, Japan. ${ }^{9}$ Division of Radiation Oncology, Shizuoka Cancer Center, 1007 Shimonagakubo, Nagaizumi Town, Shizuoka 411-8777, Japan. ${ }^{10}$ Department of Radiology, Hamamatsu University School of Medicine, 1-20-1 Handayama Higashi-ku, Hamamatsu city, Shizuoka 431-3192, Japan. ${ }^{11}$ Department of Radiology, Chiba University Graduate School of Medicine, 1-8-1 Inohana, Chiba 260-8677, Japan. ${ }^{12}$ Department of Radiation Oncology, National Cancer Center Hospital, 5-1-1 Tsukiji, Chuo-ku, Tokyo 104-0045, Japan. ${ }^{13}$ Department of Radiation Oncology, Tokyo Metropolitan Komagome Hospital, 18-22, Honkomagome 3chome, Bunkyo-ku, Tokyo 113-8677, Japan.

${ }^{14}$ Department of Radiology, Kyorin University School of Medicine, 6-20-2 Shinkawa, Mitaka-shi, Tokyo 181-8611, Japan. ${ }^{15}$ Department of Radiology, Nagoya City University Graduate School of Medical Sciences, Kawasumi, Mizuho-cho, Mizuho-ku Nagoya, Aichi 467-8601, Japan. ${ }^{16}$ Department of Radiology, Kyoto Prefectural University of Medicine, Kajii-cho, KawaramachiHirokoji, Kamigyo-ku, Kyoto 602-8566, Japan. ${ }^{17}$ Department of Radiology, Okayama University, 2-5-1 Shikata-cho, Kita-ku, Okayama-shi, Okayama 700-8558, Japan. ${ }^{18}$ Department of Radiation Oncology, Yamagata University, 2-2-2 lida-Nishi, Yamagata-shi, Yamagata 990-9585, Japan. ${ }^{19}$ Department of Radiation Oncology, Kinki University Faculty of Medicine, 377-2, OhnoHigashi, Osaka-Sayama, Osaka 589-8511, Japan.

Received: 21 December 2012 Accepted: 23 March 2013 Published: 1 April 2013 


\section{References}

1. Park SW, Park YS, Chung JB, Kang JK, Kim KS, Choi JS, Lee WJ, Kim BR, Song SY: Patterns and relevant factors of tumor recurrence for extrahepatic bile duct carcinoma after radical resection. Hepatogastroentelogy 2004 51:1612-1618

2. Matsuda T, Marugame T, Kamo K, Katanoda K, Ajiki W, Sobue T, Japan Cancer Surveillance Research Group: Cancer incidence and incidence rates in Japan in 2006: based on data from 15 population-based cancer registries in the monitoring of cancer incidence in Japan (MCIJ) project. Jpn J Clin Oncol 2012, 42:139-147.

3. Bonet Beltràn M, Allal AS, Gich I, Solé JM, Carrió I: Is adjuvant radiotherapy needed after curative resection of extrahepatic biliary tract cancers? A systematic review with a meta-analysis of observational studies. Cancer Treat Rev 2012, 38:111-119.

4. Shinohara ET, Mitra N, Guo M, Metz JM: Radiotherapy is associated with improved survival in adjuvant and palliative treatment of extrahepatic cholangiocarcinomas. Int J Radiat Oncol Biol Phys 2009, 74:1191-1198.

5. Nishimura Y, Koike R, Ogawa K, Sasamoto R, Murakami Y, Itoh Y, Negoro Y, Itasaka S, Sakayauchi T, Tamamoto T: Clinical practice and outcome of radiotherapy for esophageal cancer between 1999 and 2003: the Japanese radiation oncology study group (JROSG) survey. Int I Clin Oncol 2012, 17:48-54.

6. Ogawa K, Ito Y, Karasawa K, Ogawa Y, Onishi H, Kazumoto T, Shibuya K, Shibuya H, Okuno Y, Nishino S, Ogo E, Uchida N, Karasawa K, Nemoto K, Nishimura Y, JROSG Working Subgroup of Gastrointestinal Cancers: Patterns of radiotherapy practice for pancreatic cancer in Japan: results of the Japanese radiation oncology study group (JROSG) survey. Int J Radiat Oncol Biol Phys 2010, 77:743-750.

7. Shibuya $\mathrm{H}$, Tsujii $\mathrm{H}$ : The structural characteristics of radiation oncology in Japan in 2003. Int J Radiat Oncol Biol Phys 2005, 62:1472-1476.

8. Teshima T, Numasaki H, Shibuya H, Nishio M, Ikeda H, Ito H, Sekiguchi K, Kamikonya N, Koizumi M, Tago M, Nagata Y, Masaki H, Nishimura T, Yamada S, Japanese Society of Therapeutic Radiology and Oncology Database Committee: Japanese structure of radiation oncology in 2005 based on institutional stratification of patterns of care study. Int J Radiat Oncol Biol Phys 2008, 72:144-152.

9. Alden ME, Mohiuddin M: The impact of radiation dose in combined external beam and intraluminal Ir-192 brachytherapy for bile duct cancer. Int J Radiat Oncol Biol Phys 1994, 28:945-951.

10. Lu JJ, Bains YS, Abdel-Wahab M, Brandon AH, Wolfson AH, Raub WA, Wilkinson CM, Markoe AM: High-dose-rate remote afterloading intracavitary brachytherapy for the treatment of extrahepatic biliary duct carcinoma. Cancer J 2002, 8:74-78.

11. Milano MT, Chmura SJ, Garofalo MC, Rash C, Roeske JC, Connell PP, Kwon $\mathrm{OH}$, Jani $\mathrm{AB}$, Heimann R: Intensity-modulated radiotherapy in treatment of pancreatic and bile duct malignancies: toxicity and clinical outcome. Int J Radiat Oncol Biol Phys 2004, 59:445-453.

12. Takamura A, Saito H, Kamada T, Hiramatsu K, Takeuchi S, Hasegawa M, Miyamoto N: Intraluminal low-dose-rate 192Ir brachytherapy combined with external beam radiotherapy and biliary stenting for unresectable extrahepatic bile duct carcinoma. Int J Radiat Oncol Biol Phys 2003, 57:1357-1365.

13. Jarnagin WR, Ruo L, Little SA, Klimstra D, D'Angelica M, DeMatteo RP, Wagman R, Blumgart LH, Fong Y: Patterns of initial disease recurrence after resection of gallbladder carcinoma and hilar cholangiocarcinoma: implications for adjuvant therapeutic strategies. Cancer 2003, 98:1689-1700.

14. Crane $\mathrm{CH}$, Macdonald KO, Vauthey JN, Yehuda P, Brown T, Curley S, Wong A, Delclos M, Charnsangavej C, Janjan NA: Limitations of conventional doses of chemoradiation for unresectable biliary cancer. Int J Radiat Oncol Biol Phys 2002, 53:969-974.

15. Brunner TB, Schwab D, Meyer T, Sauer R: Chemoradiation may prolong survival of patients with non-bulky unresectable extrahepatic biliary carcinoma. A retrospective analysis. Strahlenther Onkol 2004, 180:751-757.

16. Shin HS, Seong J, Kim WC, Lee HS, Moon SR, Lee IJ, Lee KK, Park KR, Suh CO, Kim GE: Combination of external beam irradiation and high-dose-rate intraluminal brachytherapy for inoperable carcinoma of the extrahepatic bile ducts. Int J Radiat Oncol Biol Phys 2003, 57:105-112.

17. Chen YX, Zeng ZC, Tang ZY, Fan J, Zhou J, Jiang W, Zeng MS, Tan YS: Determining the role of external beam radiotherapy in unresectable intrahepatic cholangiocarcinoma: a retrospective analysis of 84 patients. BMC Cancer 2010, 10:492.

18. Nelson JW, Ghafoori AP, Willett CG, Tyler DS, Pappas TN, Clary BM, Hurwitz HI, Bendell JC, Morse MA, Clough RW, Czito BG: Concurrent chemoradiotherapy in resected extrahepatic cholangiocarcinoma. Int J Radiat Oncol Biol Phys 2009, 73:148-153.

19. Czito BG, Anscher MS, Willett CG: Radiation therapy in the treatment of cholangiocarcinoma. Oncology (Williston Park) 2006, 20:873-884.

20. Valle J, Wasan H, Palmer DH, Cunningham D, Anthoney A, Maraveyas A, Madhusudan S, Iveson T, Hughes S, Pereira SP, Roughton M, Bridgewater J, ABC-02 Trial Investigators: Cisplatin plus gemcitabine versus gemcitabine for biliary tract cancer. N Engl J Med 2010, 362:1273-1281.

doi:10.1186/1748-717X-8-76

Cite this article as: Isohashi et al:: Patterns of radiotherapy practice for biliary tract cancer in Japan: results of the Japanese radiation oncology study group (JROSG) survey. Radiation Oncology 2013 8:76.

\section{Submit your next manuscript to BioMed Central and take full advantage of:}

- Convenient online submission

- Thorough peer review

- No space constraints or color figure charges

- Immediate publication on acceptance

- Inclusion in PubMed, CAS, Scopus and Google Scholar

- Research which is freely available for redistribution 